\title{
Mutagenesis of Putative Acylation Sites Alters Function, Localization, and Accumulation of a Gi $\alpha$ Subunit of the Chestnut Blight Fungus Cryphonectria parasitica
}

\author{
Shaojian Gao and Donald L. Nuss \\ Center for Agricultural Biotechnology, University of Maryland Biotechnology Institute, University of Mary- \\ land, College Park 20742-3351 U.S.A. \\ Accepted 30 June 1998.
}

\begin{abstract}
Targeted disruption of cpg-1, a gene encoding the G protein Gi $\alpha$ subunit, CPG-1, in the chestnut blight fungus, Cryphonectria parasitica, results in reduced mycelial growth, reduced orange pigmentation, loss of virulence, loss of asexual sporulation, and female infertility. We report the development of a complementation system for cpg-1 null mutants and its use to evaluate the in vivo consequences of mutating conserved putative CPG-1 myristoylation (G2) and palmitoylation (C3) sites. Independent mutations of the two putative acylation sites differentially altered complex fungal biological processes, including virulence, and modified CPG-1 membrane association. Results of combined Northern (RNA) and Western (immunoblot) analysis also indicated a role for lipid modification in post-transcriptional regulation of CPG-1 accumulation.
\end{abstract}

Additional keywords: fungal virulence.

Heterotrimeric GTP-binding proteins are a family of regulatory complexes that play an essential role in the response of eukaryotic cells to a variety of external stimuli (Gilman 1987). The importance of $\mathrm{G}$ protein signaling in regulating diverse biological processes in filamentous fungi has only recently been demonstrated, following the cloning and disruption of a number of fungal $\mathrm{G} \alpha$ subunit genes (Turner and Borkovich 1993; Choi et al. 1995; Kozak et al. 1995; Ivey et al. 1996; Gao and Nuss 1996; Regenfelder et al. 1997; Liu and Dean 1997; Alspaugh et al. 1997). Results of these studies have also suggested the existence of a fungal $\mathrm{G}$ protein $\alpha$ subunit subfamily composed of homologues of the mammalian Gi $\alpha$ subunit genes (Ivey et al. 1996). Four of these family members-gnal from Neurospora crassa (Turner and Borkovich 1993), cpg-1 from Cryphonectria parasitica (Choi et al.

Corresponding author: Donald L. Nuss, University of Maryland Biotechnology Institute, Center for Agricultural Biotechnology, Plant Sciences Building, Room 5115C, College Park 20742-4450 U.S.A.; Telephone: 1-301-405-0334; Fax: 1-301-314-9075; E-mail: nuss@umbi.umd.edu
1995), fadA from Aspergillus nidulans (Yu et al. 1996), and $m a g B$ from Magnaporthe grisea (Liu and Dean 1997)—were shown to be highly conserved (approximately $98 \%$ identity at the predicted amino acid level), suggesting that they may play similar roles in important physiological processes in these filamentous fungi. In this regard, recent targeted gene disruption studies have revealed that members of this subfamily regulate a wide range of biological functions that can include the rate of mycelial growth, pigmentation, asexual sporulation, and female fertility. Significantly, the disruption of $C$. parasitica cpg- 1 also resulted in loss of fungal virulence, providing one of the first indications for an involvement of $G$ proteinmediated signal transduction in fungal pathogenicity (Gao and Nuss 1996). A role for $m a g B$ in $M$. grisea virulence was also recently reported (Liu and Dean 1997).

To further examine fungal Gi $\alpha$-mediated signal transduction, we have developed a system for complementing a characterized cpg-1 null mutant, disruptant G1-1 (Gao and Nuss 1996), that had been generated by targeted disruption of the cpg-1 gene in C. parasitica EP155 (ATCC 38755). A general cpg-1 complementation vector, pGPEX1, was constructed from previously characterized $\mathrm{cpg}-\mathrm{l}$ genomic clones (Choi et al. 1995) in a pUC19 genetic background (Fig. 1). The promoter region (cpg-1p) extended 1,202 nucleotides upstream from the cpg- 1 translational initiation site, while the cpg-1 terminator region (cpg-1t) extended 889 nucleotides downstream of the translational termination codon TAG. A fragment containing multiple unique cloning sites (KpnI, SmaI, and $X b a \mathrm{I}$ ) was generated by polymerase chain reaction (PCR) and introduced between cpg-1p and cpg-1t. Wild-type and mutant cpg- 1 cDNAs generated by PCR were directionally cloned into the $K p n I$ and $X b a \mathrm{I}$ sites to generate complementation transformation vectors. Transformation (Churchill et al. 1990) of G1-1 spheroplasts with vector pGPEX1 containing a wild-type cpg- 1 coding domain resulted in complete restoration of the wild-type C. parasitica EP155 phenotype (Fig. 2, CPG-1c). Complemented transformants were readily obtained by selecting for mycelial colonies that quickly emerged through the hygromycin B-containing regeneration overlay medium, presumably due to the increased growth phenotype 
relative to that of the G1-1 cpg-1 disruptant (Gao and Nuss 1996 and Fig. 2 [compare EP155 with G1-1]).

Co- and post-translational lipid modifications of $\mathrm{G}$ protein subunits significantly affect subcellular localization and protein-protein interactions (Gallego et al. 1992; Jones et al. 1990; Linder et al. 1991, 1993). For G $\alpha$ subunits these modifications include covalent attachment of $\mathrm{C} 14$ saturated acid myristate and/or C16 saturated palmitic acid to $\mathrm{N}$-terminal glycine and cysteine residues, respectively. Previous studies have revealed that mutations of the myristoylation and palmitoylation sites of mammalian $\mathrm{Gi} \alpha, \mathrm{G} \alpha 0$, and $\mathrm{G} \alpha \mathrm{z}$ subunits altered their ability to associate with the cell membrane, and greatly reduced their affinity for $\beta \gamma$ dimers and effector molecules (Mumby et al. 1994; Linder et al. 1993; Galbiati et al. 1994; Wedegaertner et al. 1995). C. parasitica CPG-1 and related members of the proposed fungal Gi $\alpha$ subfamily share

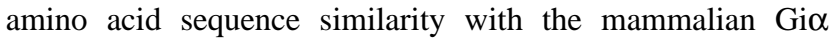
subunits, including the conservation of putative myristoylation

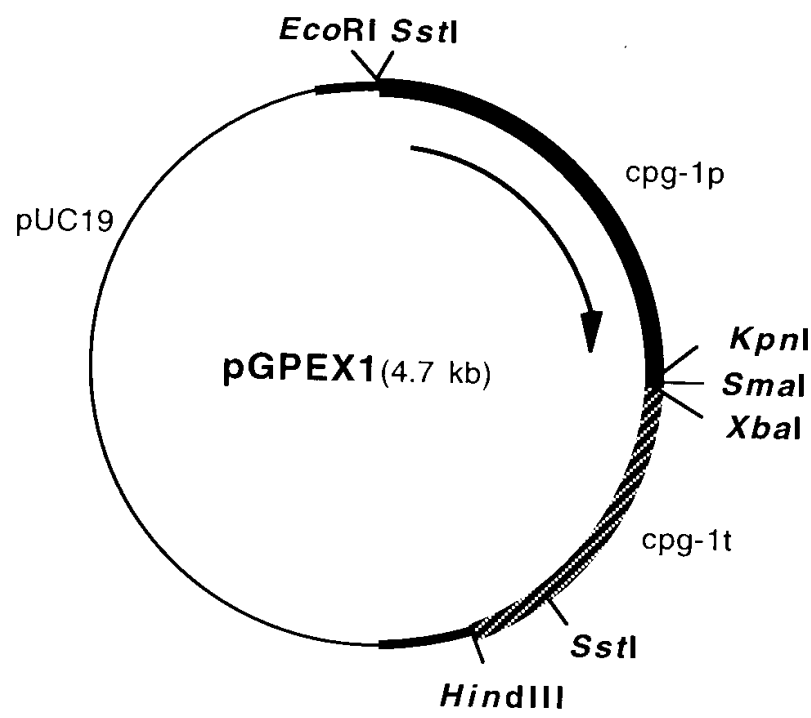

Fig. 1. Schematic map of the basic complementation vector pGPEX1. Major elements of plasmid are described in text. Wild-type and variant copies of cpg-1 coding region were generated by polymerase chain reaction (PCR) from a cpg-1 cDNA clone with a forward primer (5'-CC GGT ACC ATG $G G T T G C$ GGA ATG AGC-3') that contained a $K p n I$ site at its $5^{\prime}$ end and a reverse primer $\left(5^{\prime}-\mathrm{CC}\right.$ TCT AGA CTA GAT CAG ACC GCA AAG-3') that contained a XbaI site. Positions within the primer sequences of initiator ATG and terminator TAG codons are indicated in bold, while terminal KpnI and $\mathrm{XbaI}$ restriction sites are underlined. N-terminal codons corresponding to putative myristoylation and palmitoylation sites, glycine residue 2 (G2) and cysteine residue 3 (C3), respectively, are italicized. PCR products were digested with both KpnI and $X b a \mathrm{I}$, then cloned into the corresponding sites of pGPEX1. The sequences flanking the inserted $c p g-1$ coding domain differed slightly from the native cpg-1 cDNA sequence by the addition of 5 nucleotides (GTACC) immediately upstream of the initiation codon ATG and a G to $T$ substitution immediately after the termination codon. As indicated in the Results (Fig. 3B), these minor alterations appeared to have no effect on cpg-1 transcript accumulation. Mutations of the $\mathrm{G} 2$ and $\mathrm{C} 3$ residues to alanines in the cpg-1 coding domain (designated as $\mathrm{G} 2 \mathrm{~A}, \mathrm{C} 3 \mathrm{~A}$, and the double mutant GCAA, respectively) were obtained by changing the corresponding codons in the forward primers (G2A: GGT $\rightarrow$ GTT; C3A: TGC $\rightarrow$ GTC; GCAA: GGT TGC $\rightarrow$ GTT GTC). PCR was performed with cloned $p f u$ DNA polymerase (Stratagene, La Jolla, CA) for 30 cycles with the following parameters: $94^{\circ} \mathrm{C}, 1 \mathrm{~min} ; 55^{\circ} \mathrm{C}, 1 \mathrm{~min}$; and $72^{\circ} \mathrm{C}$, $2 \mathrm{~min}$, and a final extension of $10 \mathrm{~min}$. The cloned wild-type and mutant amplicons were sequenced in their entirety prior to use.
(G2) and palmitoylation (C3) sites. Consequently, we took advantage of the G1-1/pGPEX1 complementation system to evaluate the importance of these two putative acylation sites for $C$. parasitica virulence and the additional complex fungal biological processes regulated through the CPG-1 signaling pathway.

The protocol of collecting mycelial colonies that were first to emerge through the hygromycin B-containing overlay medium also proved effective for selection after transformation of G1-1 with pGPEX1 carrying cpg- 1 coding domains that were mutated by alanine substitutions at the putative myristoylation site (glycine $2[\mathrm{G} 2 \mathrm{~A}]$ ), the putative palmitoylation site (cysteine $3[\mathrm{C} 3 \mathrm{~A}]$ ), or both sites (GCAA). Twenty transformants were selected for each transformation construct. In each case, approximately $80 \%$ of the transformants exhibited similar stable morphological characteristics. After singlesporing, or several rounds of subculturing, two independent transformants for each transformation vector were chosen for analysis by Southern and Northern (RNA) blotting. As previously reported (Gao and Nuss 1996), hybridization of SstIdigested genomic DNA with a probe consisting of the cpg-1 coding domain revealed that a single $3.2-\mathrm{kb}$ band present in untransformed strain EP155 was replaced by a $4.5-\mathrm{kb}$ band in the disruptant G1-1 as a result of targeted insertion of a hygromycin B phosphotransferase cassette (Fig. 3A). Each of the transformants that were complemented with a wild-type or a mutated cpg- 1 cDNA copy, with the exception of one double mutant, contained an additional, expected 3.1-kb hybridizing band corresponding to the integrated cDNA. The presence of a 4.1- rather than a 3.1-kb hybridizing band (Fig. 3A) for the one exception is likely due to the loss of one of the vector Sst I sites during heterologous recombination by the pGPEX1complementation plasmid. The fact that the integrated cpg-1 cDNA bands and the 4.5-kb disrupted cpg- 1 band were of similar signal intensity, coupled with the absence of additional strong signals, suggested that each of the transformants selected for further characterization contained a single integrated copy of the cpg-1 cDNA. Importantly, Northern blot analysis showed that strain EP155 and the complementation transformants all accumulated a similar amount of cpg- 1 transcripts (Fig. 3B).

The fact that the complete EP155 wild-type phenotype was restored by complementation with pGPEX1 containing wildtype cpg-1 cDNA (Fig. 2, Table 1; and data not shown), coupled with the observation that all complemented transformants contained wild-type levels of cpg-1 mRNA, indicates that (i) the wild-type cpg- 1 cDNA used in this study encoded completely functional CPG-1 and (ii) the pGPEX1 vector contains all information required for appropriately regulated synthesis of cpg- 1 transcripts from an ectopic integration site. The observation that an ectopic copy of cpg- 1 cDNA under the control of the cpg-1 promoter can restore the wild-type phenotype in a cpg- 1 disruptant also confirms previous conclusions (Gao and Nuss 1996) that an intact CPG-1 signaling pathway is required for optimal $C$. parasitica mycelial growth, orange pigmentation, sporulation, and virulence.

Transformants containing cpg- 1 cDNA mutated at the putative $\mathrm{N}$-terminal palmitoylation site (C3A) also exhibited an increase in radial colony growth to near wild-type levels (Fig. 2). In contrast, transformation with cpg- 1 cDNA mutated at the putative myristoylation site $(\mathrm{G} 2 \mathrm{~A})$ resulted in only a very 
slight increase in radial growth. However, even this very modest increase resulted in early emergence through the overlay. Transformants containing the two different mutant cpg- 1 cDNAs also exhibited differences in levels of conidiation and orange pigmentation. The G2A transformants produced an apparently normal level of orange pigments, which was higher than observed for G1-1, but failed, like G1-1, to produce any conidia even with an extended incubation time of up to 1 month. In contrast, the $\mathrm{C} 3 \mathrm{~A}$ transformants did produce spores at a moderate level (approximately $10^{5}$ versus approximately $10^{9}$ for wild-type strain EP155), but exhibited very little restoration of orange pigmentation.

Interpretation of the effect of mutation of the putative myristoylation site is potentially complicated by a report that the G2A mutation has a negative effect on palmitoylation (Galbiati et al. 1994). Thus, some of the phenotypic characteristics observed for the G2A transformants may be due to the combined effect of reduced myristoylation and palmitoy- lation. In this regard, transformants containing cpg-1 cDNA mutated at both putative acylation sites (GCAA) did closely resemble the G2A transformants in colony morphology (Fig. $2)$, sporulation levels (data not shown), and orange pigmentation (Fig. 2), but grew slightly slower (Fig. 2).

Targeted disruption of cpg-1 was previously shown to abolish $C$. parasitica virulence on American chestnut tissue (Gao and Nuss 1996). As shown in Table 1, mutations in the putative cpg-1 myristoylation and palmitoylation sites also had different impacts on the ability to restore virulence. Transformation with the $\mathrm{C} 3 \mathrm{~A}$ mutant restored virulence to nearly the same level as was observed for transformants containing unmutated cpg- 1 cDNA (CPG-1c) or wild-type strain EP155. In contrast, transformation with the G2A mutant increased virulence only slightly, to a level similar to that exhibited by hypovirus-infected EP713 (ATCC 52571) (Table 1). Transformation with the double-site mutant GCAA resulted in even less of an increase in virulence than exhibited by the G2A

\section{$\begin{array}{lll}\text { EP155 CPG-1C } & \text { C3A }\end{array}$}

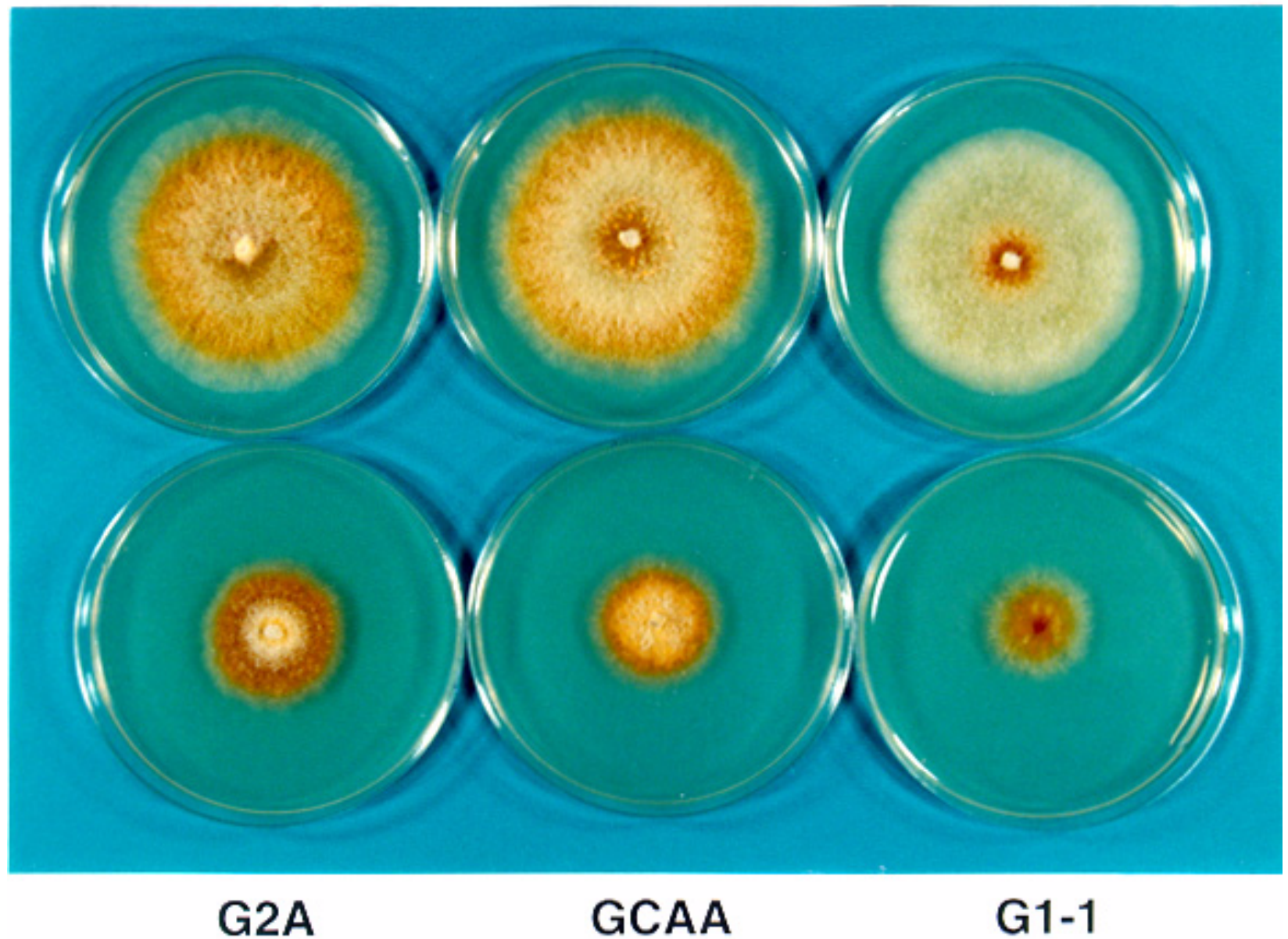

Fig. 2. Colony morphology of wild-type strain EP155, the cpg-1 disruption mutant G1-1, and the G1-1 complementation transformants containing wildtype cpg- 1 cDNA (CPG-1c), cpg- 1 cDNA containing the glycine to alanine substitution (G2A), cpg- 1 cDNA containing the cysteine to alanine substitution (C3A), and cpg-1 cDNA containing the glycine/cysteine to alanines double-mutant (GCAA). Spheroplasts prepared from the $c p g$-1-disrupted Cryphonectria parasitica strain G1-1 were transformed following procedures described by Churchill et al. (1990). Transformants were first transferred to potato dextrose agar (PDA) containing $50 \mu \mathrm{g}$ of hygromycin B per $\mathrm{ml}$ and then subcultured on PDA. Those transformants that sporulated on PDA were purified to nuclear homogeneity by isolating colonies derived from single conidia. Those transformants that failed to sporulate were subcultured several rounds on PDA to confirm morphological stability. Colonies were incubated at room temperature (approximately $23^{\circ} \mathrm{C}$ ) for 7 days. Comparative examinations of radial colony growth, orange pigmentation, and asexual sporulation were performed as previously described (Hillman et al. 1990). 
transformants, suggesting that in the case of virulence, mutations of the two putative acylation sites produce an additive effect.

It is intriguing that while cpg- 1 disruption results in complete loss of conidiation and virulence and a severe reduction in growth and pigmentation, independent mutations of the putative acylation sites result in intermediate reductions in a subset of these processes. This suggests that while each of these physiological processes requires an intact CPG-1 signaling pathway for optimal execution, they appear to be differentially affected by alterations in that pathway. In considering these differential responses, it is important to remember that only two G $\alpha$ subunit genes, cpg-1 and cpg-2 (Choi et al. 1995; Gao and Nuss 1996), and a single G $\beta$ subunit gene, cpgb-1 (Kasahara and Nuss 1997), have been identified for $C$. parasitica. Disruption of cpg-2 results in little phenotypic change while cpg-1 disruption has the severe consequences noted above (Gao and Nuss 1996). Thus, as is observed for G

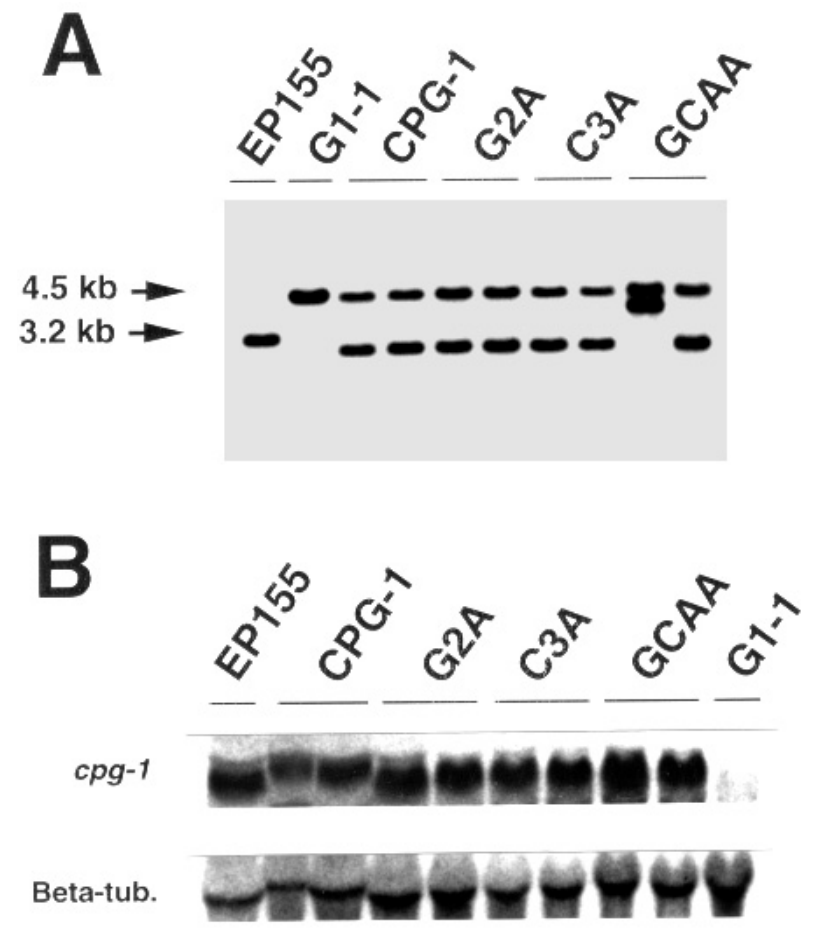

Fig. 3. Southern and Northern (RNA) blot analyses for cpg-1 in the wild-type strain EP155, the cpg-1 disruptant G1-1, and the G1-1 complementation transformants containing wild-type cpg-1 cDNA (CPG-1c), or cpg-1 cDNA mutated at the putative myristoylation/palmitoylation sites (G2A, C3A, and GCAA). Nucleic acid preparation and Southern and Northern blot analyses were performed as previously described (Gao and Nuss 1996). Single-copy integration transformants were selected based on Southern analyses and used for all subsequent analyses. A, Southern blot analysis of the integrated cpg-1 cDNA in the complementation transformants. Total nucleic acid $(10 \mu \mathrm{g})$ from each strain was digested with SstI, separated on a $0.8 \%$ agarose gel, and transferred to a Hybond-N membrane. The membrane blot was probed with a fulllength, ${ }^{32}$ P-labeled cpg-1 cDNA. Sizes of expected hybridization bands are indicated at left. B, Northern hybridization analysis of cpg-1 transcript accumulation in the complementation transformants. Total RNA $(10 \mu \mathrm{g})$ was loaded in each well, separated in a formaldehyde-1.4\% agarose gel, and transferred to a Hybond-N membrane. The blot was hybridized with a full-length, ${ }^{32} \mathrm{P}$-labeled $c p g-1$ cDNA. After autoradiography, the blot was stripped and rehybridized with a $C$. parasitica $\beta$ tubulin cDNA probe (Choi et al. 1992). proteins in other eukaryotes (Gilman 1987), CPG-1 is likely to be involved in the transduction of a variety of different stimuli and to serve as a common component of multiple regulatory pathways that may also be subject to cross talk. Accordingly, it is not unreasonable to expect that the different CPG-1regulated processes, ranging from virulence to conidiation, would exhibit different sensitivities to modulations in the output level of the CPG-1 signaling pathway.

Mutation of the N-terminal myristoylation and palmitoylation sites has been reported to alter the membrane association properties for some $\mathrm{G}$ protein $\alpha$ subunits (e.g., Mumby et al. 1990; Linder et al. 1991; Gallego et al. 1992; Galbiati et al. 1994). Consistent with these reports, mutation of putative CPG-1 acylation sites was found to alter subcellular distribution. Unexpectedly, mutation of the putative myristolyation site also resulted in a significant increase in CPG-1 accumulation. As indicated in Figure 4A, the total levels of CPG-1 accumulation in the wild-type strain EP155, the CPG-1c control transformant, and the palmitoylation site mutants $\mathrm{C} 3 \mathrm{~A}$ were similar. However, CPG-1 accumulation levels were consistently observed to be substantially increased for both the putative myristoylation site mutants G2A and the double-site mutants GCAA (Fig. 4A). Examination of the distribution of CPG-1 between membrane (P) and cytosolic (S) fractions in the wild-type strain EP155 and the CPG-1c complemented strain confirmed that this member of the fungal Gi $\alpha$ family was found predominantly associated with the membrane fraction (Fig. 4B). Mutation of the putative palmitoylation site (C3A) altered this distribution, resulting in an apparent relative increase in cytosolic CPG-1 and a concomitant decrease in membrane-associated CPG-1 (Fig. 4B). This analysis also indicated that the elevation in CPG-1 accumulation observed for the myristoylation mutant G2A and the double-site mutant GCAA correlated with a large increase in cytosolic CPG-1. Since cpg- 1 mRNA accumulated to similar levels in wild-type and mutant transformant strains (Fig. 3B), the unexpected increase in CPG-1 levels observed in the G2A and GCAA mutant transformants suggests a possible role for the putative myristoylation site in the post-transcriptional regulation of CPG-1 accumulation.

Structure/function studies for mammalian Gi $\alpha$ subunits generally rely on transient expression in heterologous tissue

Table 1. Mean canker areas induced on American chestnut stems by virulent Cryphonectria parasitica strain EP155 and the cpg-1 disruptant G1-1 complemented with wild-type cpg-1 cDNA (CPG-1c) and cpg-1 cDNAs mutated at the putative myristoylation site $(\mathrm{G} 2 \mathrm{~A})$, the putative palmitoylation site (C3A), or at both sites (GCAA)

\begin{tabular}{lc}
\hline Strains and mutants & Mean canker area $\pm \mathbf{S D}\left(\mathbf{c m}^{\mathbf{2}}\right)^{\mathbf{a}}$ \\
\hline EP155 & $9.87 \pm 2.46$ \\
CPG-1c & $8.40 \pm 2.33$ \\
C3A & $8.48 \pm 2.23$ \\
G2A & $1.89 \pm 0.35$ \\
GCAA & $0.63 \pm 0.42$ \\
EP713 & $1.89 \pm 0.30$ \\
\hline
\end{tabular}

${ }^{a}$ Mean canker areas for strains EP155 and EP713 were determined 14 days after inoculation based on six replications (Jaynes and Elliston 1980; Choi and Nuss 1992). Values for G1-1 complementation transformants CPG-1c, G2A, C3A, and GCAA are based on six replications for two independent transformants of each. As reported previously (Gao and Nuss 1996), the cpg-1 disruptant G1-1 failed to expand on chestnut tissue. 

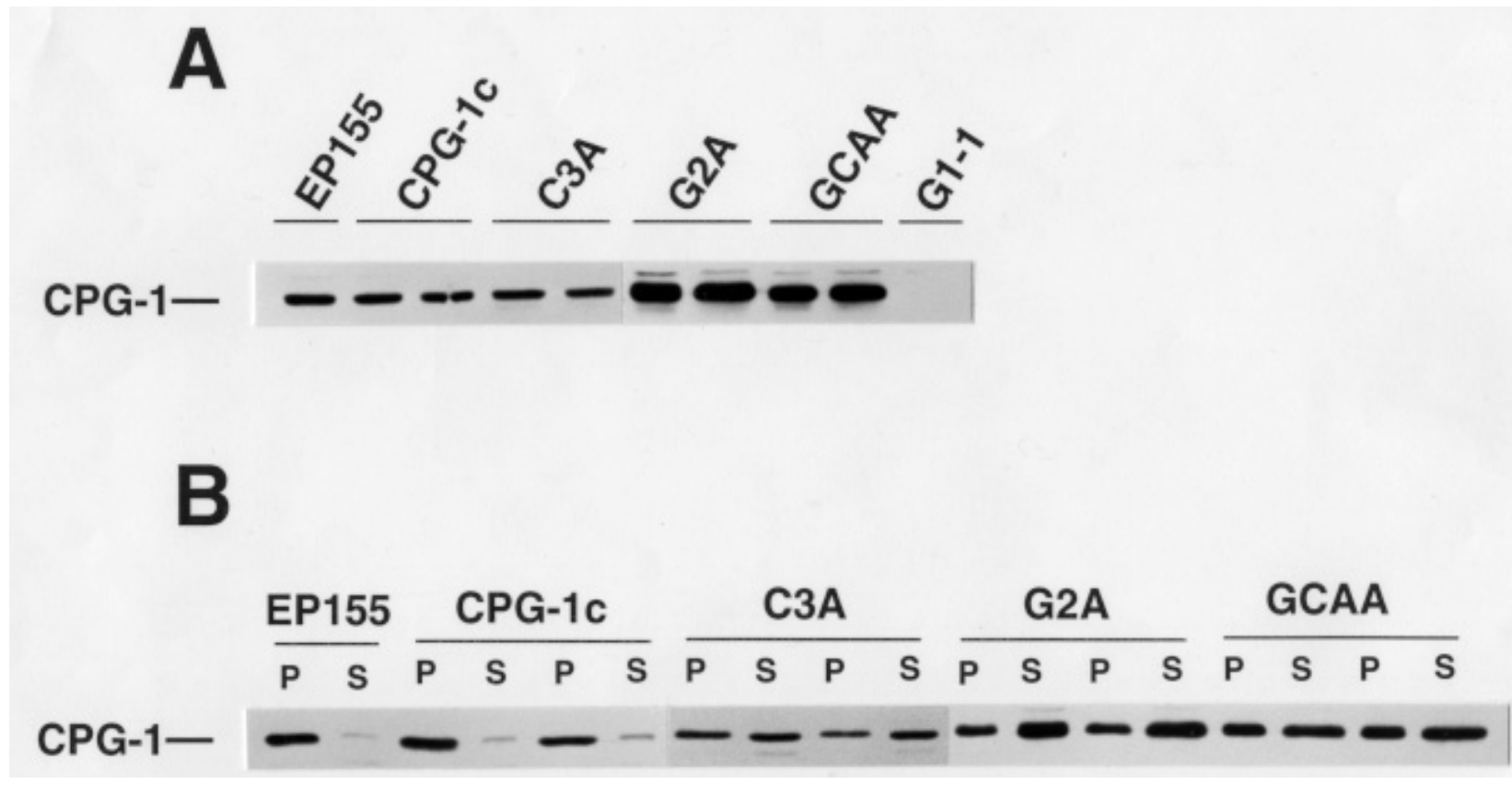

Fig. 4. Western blot (immunoblot) analysis of CPG-1 levels in strain EP155, the cpg-1 disruptant G1-1, and the G1-1 complementation transformants containing cDNAs for wild-type cpg-1 (CPG-1c) and the putative myristoylation/palmitoylation sites mutants (G2A, C3A, and GCAA). A, Total CPG-1 levels in each strain. B, Subcellular localization of CPG-1. Supernatant after centrifugation at 150,000 $\times g$ for 30 min was designated as the soluble fraction (S). Pellet fraction was resuspended in an equal volume of extraction buffer and designated as the membrane fraction (P). Fungal colonies used to prepare extracts for Western blot analysis of CPG-1 were grown on cellophane-covered potato dextrose agar (cellophane/PDA) plates incubated on the bench-top at room temperature for 7 days. Harvested mycelium (approximately $0.5 \mathrm{~g}$ ) was ground into a powder in liquid nitrogen with a mortar and pestle and transferred into a $1.5-\mathrm{ml}$ microcentrifuge tube. The mycelial powder was then suspended in $0.6 \mathrm{ml}$ of extraction buffer $(0.1 \mathrm{M}$ Tris-HCl [pH8.0], $10 \mathrm{mM}$ dithiothreitol, $1 \mathrm{mM}$ EDTA, $0.5 \mathrm{mM}$ EGTA, $1 \mathrm{mM}$ phenylmethylsulfonyl fluoride, $10 \mu \mathrm{g}$ of leupeptin per ml, and $10 \mu \mathrm{g}$ of aprotinin per $\mathrm{ml}$ ) on ice and incubated for $10 \mathrm{~min}$. The extract was cleared by centrifugation twice at $4,000 \times \mathrm{g}$ for $5 \mathrm{~min}$ at $4^{\circ} \mathrm{C}$ each. Protein concentration of the supernatants was determined by the Bradford micro assay (Bradford 1976) with the Bio-Rad (Hercules, CA) protein assay kit and bovine serum albumin as standard. Western blot analysis was performed by resolving $30 \mu \mathrm{g}$ of proteins on a $12 \%$ sodium dodecyl sulfate-polyacrylamide gel electrophoresis (SDS-PAGE) gel followed by transfer of separated proteins to nitrocellulose with a Sartoblot II (Sartorium Filters, Hayward, CA) semi-dry blotter and probing with affinity-purified anti-CPG-1 polyclonal antibody. CPG-1 was detected with the ECL Western detection system with anti-rabbit IgG secondary antibodies conjugated with horseradish peroxidase (Amersham, Arlington Heights, IL) (Choi et al. 1995). In the case of subcellular localization studies, the crude extracts $\left(100 \mu \mathrm{g}\right.$ ) were fractionated by centrifugation at $150,000 \times \mathrm{g}$ for $30 \mathrm{~min}$ at $4^{\circ} \mathrm{C}$ in a TL-100 ultracentrifuge (Beckman Instruments, Fullerton, CA).

culture cell lines or on the addition of in vitro synthesized proteins to membrane preparations. These studies often focus on molecular interactions and catalytic activities rather than effects on complex physiological processes at the organismal level. As noted by Ivey et al. (1996), Gi $\alpha$ subunit homologue genes appear to be absent in the Saccharomyces cerevisiae or Schizosaccharomyces pombe genomes, limiting the utility of these genetically tractable systems for studying Gi $\alpha$ subunit function. Additionally, Kasahara and Nuss (1997) recently reported that the amino acid sequence of $C$. parasitica CPGB1 , the only currently characterized filamentous fungal $\mathrm{G}$ protein $\beta$ subunit, is much more closely related to a human $G \beta$ than it is to the $S$. cerevisiae homologue Ste4p. Thus, the plant-pathogenic fungus $C$. parasitic would appear to provide certain advantages over other commonly studied lower eukaryotes as an experimental system with which to examine detailed regulatory mechanisms underlying Gi $\alpha$ accumulation and function.

\section{ACKNOWLEDGMENTS}

This work was funded by a grant from the National Institutes of Health, GM55981 to D. L. N.

\section{LITERATURE CITED}

Alspaugh, J. A., Perfect, J. R., and Heitman, J. 1997. Cryptococcus neoformans mating and virulence are regulated by the G-protein $\alpha$ subunit GPA1 and cAMP. Genes Dev. 11:3206-3216.

Bradford, M. M. 1976. A rapid and sensitive method for quantitation of microgram quantities of protein utilizing the principle of protein-dye binding. Anal. Biochem. 72:248-254.

Choi, G. H., Chen, B., and Nuss, D. L. 1995. Virus-mediated or transgenic suppression of a G-protein $\alpha$ subunit and attenuation of fungal virulence. Proc. Natl. Acad. Sci. USA 92:305-309.

Choi, G. H., Larson, T. G., and Nuss, D. L. 1992. Molecular analysis of the laccase gene from the chestnut blight fungus and selective suppression of its expression in an isogenic hypovirulent strain. Mol. Plant-Microbe Interact. 5:119-128.

Choi, G. H., and Nuss, D. L. 1992. Hypovirulence of chestnut blight fungus conferred by an infectious viral cDNA. Science 257:800-803.

Churchill, A. C. L., Ciufetti, L. M., Hansen, D. R., Van Etten, H. D., and Van Alfen, N. K. 1990. Transformation of the fungal pathogen Cryphonectria parasitica with a variety of heterologous plasmids. Curr. Genet. 17:25-31.

Galbiati, M., Guzzi, F., Magee, A. I., Milligan, G., and Parenti, M. 1994. N-terminal fatty acylation of the $\alpha$-subunit of the G-protein Gi1: Only the myristoylated protein is a substrate for palmitoylation. Biochem. J. 303:697-700.

Gallego, C., Gupta, S. K., Winitz, S., Eisfelder, B. J., and Johnson, G. L. 1992. Myristoylation of the Goi2 polypeptide, a G protein $\alpha$ subunit, is required for its signaling and transformation functions. Proc. Natl. 
Acad. Sci. USA 89:9695-9699.

Gao, S., and Nuss, D. L. 1996. Distinct roles for two G protein $\alpha$ subunits in fungal virulence, morphology, and reproduction revealed by targeted gene disruption. Proc. Natl. Acad. Sci. USA 93:14122-14127.

Gilman, A. G. 1987. G proteins: Transducers of receptor-generated signals. Annu. Rev. Biochem. 56:615-649.

Hillman, B. I., Shapira, R., and Nuss, D. L. 1990. Hypovirulenceassociated suppression of host functions in Cryphonectria parasitica can be partially relieved by high light intensity. Phytopathology 80:950-956.

Ivey, F. D., Hodge, P. N., Turner, G. E., and Borkovich, K. A. 1996. The $\mathrm{G} \alpha$ homologue gna-1 controls multiple differentiation pathways in Neurospora crassa. Mol. Biol. Cell. 7:1283-1297.

Jaynes, R. A., and Elliston, J. F. 1980. Pathogenicity and canker control by mixtures of hypovirulent strains of Endothia parasitica in American chestnut. Phytopathology 70:453-456.

Jones, T. L., Simonds, W. F., Merendino, J. J., Jr., and Brann, M. R. 1990. Myristoylation of an inhibitory GTP-binding protein $\alpha$ subunit is essential for its membrane attachment. Proc. Natl. Acad. Sci. USA 87:568-572.

Kasahara, S., and Nuss, D. L. 1997. Targeted disruption of a fungal Gprotein $\beta$ subunit gene results in increased vegetative growth but reduced virulence. Mol. Plant-Microbe Interact. 10:984-993.

Kozak, K. R., Foster, L. M., and Ross, I. K. 1995. Cloning and characterization of a $\mathrm{G}$ protein $\alpha$-subunit-encoding gene from the basidiomycete, Coprinus congregatus. Gene 163:133-137.
Linder, M. E., Middleton, P, Helper, J. R., Taussig, R., and Gilman, A. G. 1993. Lipid modification of $\mathrm{G}$ proteins: $\alpha$ subunits are palmitoylated. Proc. Natl. Acad. Sci. USA 90:3675-3679.

Linder, M. E., Pang, I., Duronio, R. J., Gordon, J. I., Sternweis, P. C., and Gilman A. G. 1991. Lipid modifications of $\mathrm{G}$ protein subunits: Myristoylation of $\mathrm{Go} \alpha$ increases its affinity for $\beta \gamma$. J. Biol. Chem. 266:4654-4659.

Liu, S., and Dean, R. A. 1997. G protein $\alpha$ subunit genes control growth, development, and pathogenicity of Magnaporthe grisea. Mol. Plant-Microbe Interact. 10:1075-1086.

Mumby, S. M., Heukeroth, R. O., Gordon, J. I., and Gilman, A. G. 1990 G-protein $\alpha$-subunit expression, myristoylation, and membrane association in COS cells. Proc. Natl. Acad. Sci. USA 87:728-732.

Mumby, S. M., Kleuss, C., and Gilman, A. G. 1994. Receptor regulation of G-protein palmitoylation. Proc. Natl. Acad. Sci. USA 91:2800-2804.

Regenfelder, E., Spellig, T., Hartmann, A., Lauenstein, S., Bolker, M. and Kahmann, R. 1997. G proteins in Ustilago maydis: Transmission of multiple signals? EMBO J. 16:1934-1942.

Turner, G. E., and Borkovich, K. A. 1993. Identification of a G protein $\alpha$ subunit from Neurospora crassa that is a member of the Gi family. J. Biol. Chem. 268:14805-14811.

Wedegaertner, P. B., Wilson, P. T., and Bourne, H. R. 1995. Lipid modifications of trimeric G proteins. J. Biol. Chem. 270:503-506.

Yu, J. H., Wieser, J., and Adams, T. H. 1996. The Aspergillus FlbA RGS domain protein antagonizes $\mathrm{G}$ protein signaling to block proliferation and allow development. EMBO J. 15:5184-5190. 